If you wish to distribute this article to others, you can order high-quality copies for your colleagues, clients, or customers by clicking here.

Permission to republish or repurpose articles or portions of articles can be obtained by following the guidelines here.

The following resources related to this article are available online at www.sciencemag.org (this information is current as of October 28, 2014 ):

Updated information and services, including high-resolution figures, can be found in the online version of this article at:

http://www.sciencemag.org/content/342/6162/1069.full.html

Supporting Online Material can be found at:

http://www.sciencemag.org/content/suppl/2013/11/06/science.1242642.DC2.html

http://www.sciencemag.org/content/suppl/2013/11/06/science.1242642.DC1.html

A list of selected additional articles on the Science Web sites related to this article can be found at:

http://www.sciencemag.org/content/342/6162/1069.full.html\#related

This article cites 112 articles, 12 of which can be accessed free:

http://www.sciencemag.org/content/342/6162/1069.full.html\#ref-list-1

This article has been cited by 4 articles hosted by HighWire Press; see:

http://www.sciencemag.org/content/342/6162/1069.full.html\#related-urls

This article appears in the following subject collections:

Planetary Science

http://www.sciencemag.org/cgi/collection/planet_sci 


\section{Chelyabinsk Airburst, Damage Assessment, Meteorite Recovery, and Characterization}

Olga P. Popova ${ }^{1}$ Peter Jenniskens, ${ }^{2,3 *}$ Vacheslav Emel'yanenko ${ }^{4}$ Anna Kartashova Eugeny Biryukov, ${ }^{5}$ Sergey Khaibrakhmanov, ${ }^{6}$ Valery Shuvalov, ${ }^{1}$ Yurij Rybnov, ${ }^{1}$ Alexandr Dudorov, ${ }^{6}$ Victor I. Grokhovsky, ${ }^{7}$ Dmitry D. Badyukov, ${ }^{8}$ Qing-Zhu Yin, ${ }^{9}$ Peter S. Gural, ${ }^{2}$ Jim Albers, ${ }^{2}$ Mikael Granvik, ${ }^{10}$ Läslo G. Evers, ${ }^{11,12}$ Jacob Kuiper, ${ }^{11}$ Vladimir Kharlamov, ${ }^{1}$ Andrey Solovyov, ${ }^{13}$ Yuri S. Rusakov, ${ }^{14}$ Stanislav Korotkiy, ${ }^{15}$ Ilya Serdyuk, ${ }^{16}$ Alexander V. Korochantsev, ${ }^{8}$ Michail Yu. Larionov, ${ }^{7}$ Dmitry Glazachev, ${ }^{1}$ Alexander E. Mayer, ${ }^{6}$ Galen Gisler, ${ }^{17}$ Sergei V. Gladkovsky, ${ }^{18}$ josh Wimpenny, ${ }^{9}$ Matthew E. Sanborn, ${ }^{9}$ Akane Yamakawa, ${ }^{9}$ Kenneth L. Verosub, ${ }^{9}$ Douglas ]. Rowland, ${ }^{19}$ Sarah Roeske, ${ }^{9}$ Nicholas W. Botto, ${ }^{9}$ Jon M. Friedrich, ${ }^{20,21}$ Michael E. Zolensky, ${ }^{22}$ Loan Le, $^{23,22}$ Daniel Ross, ${ }^{23,22}$ Karen Ziegler, ${ }^{24}$ Tomoki Nakamura, $^{25}$ Insu Ahn, ${ }^{25}$ Jong Ik Lee, ${ }^{26}$ Qin Zhou, ${ }^{27,28}$ Xian-Hua Li, ${ }^{28}$ Qiu-Li Li, ${ }^{28}$ Yu Liu, ${ }^{28}$ Guo-Qiang Tang, ${ }^{28}$ Takahiro Hiroi, $^{29}$ Derek Sears, ${ }^{3}$ Ilya A. Weinstein, ${ }^{7}$ Alexander S. Vokhmintsev, ${ }^{7}$ Alexei V. Ishchenko, ${ }^{7}$ Phillipe Schmitt-Kopplin, ${ }^{30,31}$ Norbert Hertkorn, ${ }^{30}$ Keisuke Nagao, ${ }^{32}$ Makiko K. Haba, ${ }^{32}$ Mutsumi Komatsu, $^{33}$ Takashi Mikouchi, ${ }^{34}$ (the Chelyabinsk Airburst Consortium)

The asteroid impact near the Russian city of Chelyabinsk on 15 February 2013 was the largest airburst on Earth since the 1908 Tunguska event, causing a natural disaster in an area with a population exceeding one million. Because it occurred in an era with modern consumer electronics, field sensors, and laboratory techniques, unprecedented measurements were made of the impact event and the meteoroid that caused it. Here, we document the account of what happened, as understood now, using comprehensive data obtained from astronomy, planetary science, geophysics, meteorology, meteoritics, and cosmochemistry and from social science surveys. A good understanding of the Chelyabinsk incident provides an opportunity to calibrate the event, with implications for the study of near-Earth objects and developing hazard mitigation strategies for planetary protection.

$(1$ helyabinsk Oblast experienced an impact that was 100 times more energetic than the recent $4 \mathrm{kT}$ of TNT-equivalent Sutter's Mill meteorite fall (1). This was the biggest impact over land since the poorly observed Tunguska impact in 1908 , for which kinetic energy estimates range from 3 to 5 (2) to 10 to 50 MT (3). From the measured period of infrasound waves circum-traveling the globe (4), an early estimate of $\sim 470 \mathrm{kT}$ was derived for Chelyabinsk (5). Infrasound data from Russia and Kazakhstan provide $570 \pm 150 \mathrm{kT}$; see supplementary materials (SM) section 1.4 (6). Spaceborne visible and near-infrared observations (7) recorded a total irradiated energy of $90 \mathrm{kT}(5,8)$, corresponding to a kinetic energy of $590 \pm 50 \mathrm{kT}$ using the calibration by Nemtchinov et al. (9). All values are uncertain by a factor of two because of a lack of calibration data at those high energies and altitudes.

The manner in which this kinetic energy was deposited in the atmosphere determined what shock wave reached the ground. Dash-camera and security camera videos of the fireball (Fig. 1) provide a light curve with peak brightness of $-27.3 \pm 0.5$ magnitude (Fig. 2) (SM section 1.2). The integrated light curve is consistent with other energy estimates if the panchromatic luminous efficiency was $7 \pm 3 \%$. Theoretical estimates under these conditions range from 5.6 to $13.2 \%(10)$.

Calibrated video observations provided a trajectory and pre-atmospheric orbit (Table 1) (SM section 1.1). The fireball was first recorded at $97-\mathrm{km}$ altitude, moving at $19.16 \pm 0.15 \mathrm{~km} / \mathrm{s}$ with entry angle $18.3 \pm 0.2^{\circ}$ with respect to the horizon, which is slightly faster than reported earlier (11). Combined with the best kinetic energy estimate, an entry mass of $1.3 \times 10^{7} \mathrm{~kg}$ (with a factor of two uncertainty) and a diameter of $19.8 \pm 4.6 \mathrm{~m}$ is derived, assuming a spherical shape and the meteoritederived density of $3.3 \mathrm{~g} / \mathrm{cm}^{3}$ based on x-ray computed tomography (SM section 4.2, table S16).

Size and speed suggest that a shock wave first developed at $90 \mathrm{~km}$. Observations show that dust formation and fragmentation started around $83 \mathrm{~km}$ and accelerated at $54 \mathrm{~km}$ (figs. S16 and S22). Peak radiation occurred at an altitude of $29.7 \pm$ $0.7 \mathrm{~km}$ at 03:20:32.2 \pm 0.1s UTC (SM section 1.1-2), at which time spaceborne sensors measured a meteoroid speed of $18.6 \mathrm{~km} / \mathrm{s}$ (5). Fragmentation left a thermally emitting debris cloud in this period, the final burst of which occurred at 27.0-km altitude (Fig. 1), with dust and gas settling at $26.2 \mathrm{~km}$ and with distinctly higher billowing above that location (fig. S22). The dust cloud split in two due to the buoyancy of the hot gas, leading to two cylindrical vortices (12).

Compared with the much larger Tunguska event $(2,3)$, Chelyabinsk was only on the threshold of forming a common shock wave around the fragments when it broke at peak brightness (SM section 1.2). Fragments were spatially isolated enough to be efficiently decelerated, avoiding the transfer of momentum to lower altitudes and resulting in less damage when the blast wave reached the ground.

\section{Damage Assessment}

In the weeks after the event, 50 villages were visited to verify the extent of glass damage. The resulting map (Fig. 3) demonstrates that the shock wave had a cylindrical component, extending furthest perpendicular to the trajectory. There was little coherence of the shock wave in the forward direction, where the disturbance was of long duration, shaking buildings and making people run outside, but causing no damage.

${ }^{1}$ Institute for Dynamics of Geospheres of the Russian Academy of Sciences, Leninsky Prospect 38, Building 1, Moscow, 119334, Russia. ${ }^{2}$ SETI Institute, 189 Bernardo Avenue, Mountain View, CA 94043, USA. ${ }^{3}$ NASA Ames Research Center, Moffett Field, Mail Stop 245-1, CA 94035, USA. ${ }^{4}$ Institute of Astronomy of the Russian Academy of Sciences, Pyatnitskaya 48, Moscow, 119017, Russia. ${ }^{5}$ Department of Theoretical Mechanics, South Ural State University, Lenin Avenue 76, Chelyabinsk, 454080, Russia. ${ }^{6}$ Chelyabinsk State University, Bratyev Kashirinyh Stree 129, Chelyabinsk, 454001, Russia. ${ }^{7}$ Institute of Physics and Technology, Ural Federal University, Mira Street 19, Yekaterinburg, 620002, Russia. ${ }^{8}$ Vernadsky Institute of Geochemistry and Analytical Chemistry of the RAS, Kosygina Street 19, Moscow, 119991, Russia. 'Department of Earth and Planetary Sciences, University of California at Davis, Davis, CA 95616, USA. ${ }^{10}$ Department of Physics, University of Helsinki, P.O. Box 64, 00014 Helsinki, Finland. ${ }^{11}$ Koninklijk Nederlands Meteorologisch Instituut, P.O. Box 201, 3730 AE De Bilt, Netherlands. ${ }^{12}$ Department of Geoscience and Engineering, Faculty of Civil Engineering and Geosciences, Delft University of Technology, P.O. Box 5048, 2600 GA Delft, Netherlands. ${ }^{13}$ Tomsk State University, Lenina Prospect 36, Tomsk, 634050, Russia. ${ }^{14}$ Research and Production Association "Typhoon," Floor 2, 7 Engels Street, Obninsk, 249032, Russia. ${ }^{15}$ Support Foundation for Astronomy "Ka-Dar," P.O. Box 82, Razvilka, 142717, Russia. ${ }^{16}$ Science and Technology Center of the Social and Youth Initiatives Organization, 3-12-63 Udal'tsova Street, Moscow, 119415, Russia. ${ }^{17}$ University of Oslo, Physics Building, Sem Saelands Vel 24, 0316 Oslo, Norway. ${ }^{18}$ Institute of Engineering Sciences Urals Branch of the Russian Academy of Sciences, Komsomolskaya Street 34, Yekaterinburg, 620049, Russia. ${ }^{19}$ Center for Molecular and Genomic Imaging, University of California, Davis, Davis, CA 95616, USA. ${ }^{20}$ Department of Earth and Planetary Sciences, American Museum of Natural History, New York, NY 10024, USA. ${ }^{21}$ Department of Chemistry, Fordham University, Bronx, NY 10458, USA. ${ }^{22}$ Astromaterials Research and Exploration Science, NASA Johnson Space Center, Houston, TX 77058, USA. ${ }^{23}$ Jacobs Technology, 2224 Bay Area Boulevard, Houston, TX 77058, USA. ${ }^{24}$ Institute of Meteoritics, University of New Mexico, Albuquerque, NM 87131-0001, USA. ${ }^{25}$ Department of Earth and Planetary Materials Science, Tohoku University, Aramaki, Aoba, Sendai, Miyagi 980-8578, Japan. ${ }^{26}$ Division of Polar Earth-System Sciences, Korea Polar Research Institute, 26 Songdomi Rae, Yeonsu-gu, Incheon 406-840, Korea. ${ }^{27}$ National Astronomical Observatories, Beijing, Chinese Academy of Sciences, Beijing 100012, China. ${ }^{28}$ State Key Laboratory of Lithospheric Evolution, Institute of Geology and Geophysics, Chinese Academy of Sciences, Beijing 100029 China. ${ }^{29}$ Department of Geological Sciences, Brown University, Providence, RI 02912, USA. ${ }^{30}$ Analytical BioGeoChemistry, Helmoltz Zentrum Muenchen, Ingoldstäter Landstrasse 1 D-85764 Obeschleissheim, Germany. ${ }^{31}$ Technical University Muenchen, Analytical Food Chemistry, Alte Akademie 10, D-85354 Freising, Germany. ${ }^{32}$ Geochemical Research Center, The University of Tokyo, 7-3-1 Hongo, Bunkyo-ku, Tokyo 113-0033, Japan. ${ }^{33}$ Waseda Institute for Advanced Study, Waseda University, 1-6-1 Nishiwaseda, Shinjuku, Tokyo 169-8050, Japan. ${ }^{34}$ Department of Earth and Planetary Science, The University of Tokyo, 7-3-1 Hongo, Bunkyo-ku, Tokyo 113-0033, Japan.

*Corresponding author. E-mail: petrus.m.jenniskens@nasa.gov 


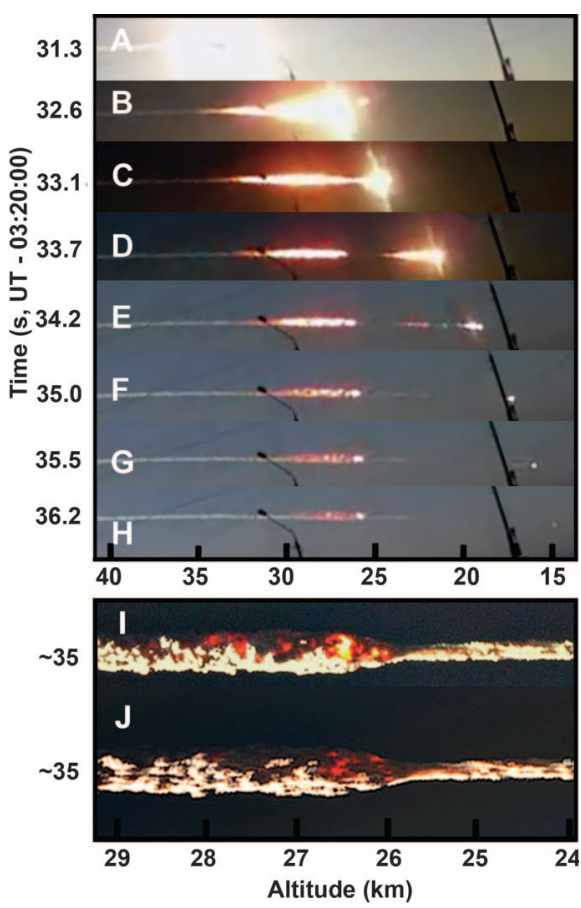

Fig. 1. Meteoroid fragmentation stages in video taken by A. Ivanov in Kamensk-Uralskiy. (A) Fireball just before peak brightness, at the moment when camera gain was first adjusted. (B) End of main disruption. (C) Onset of secondary disruption. (D) End of secondary disruption; main debris cloud continues to move down. (E) Two main fragments remain. (F) Single fragment remains. (G) Thermally emitting debris cloud at rest with atmosphere. (H) Final fragment continues to penetrate. Meteor moved behind distant lamp posts. (I and J) Detail of the thermal emission from a photograph by Mr. Dudarev (I) and M. Ahmetvaleev (J), after sky subtraction with high-pass filter and contrast enhancement. Altitude scale is uncertain by $\pm 0.7 \mathrm{~km}$.

The strength of this shock wave on the ground was modeled (SM section 2.4), assuming that an overpressure of $\Delta p>500 \mathrm{~Pa}$ was required (13). A 520-kT event, with detonations spread over altitudes ranging from 34 to $27 \mathrm{~km}$ and 24 to $19 \mathrm{~km}$, would cause damage out to a distance of $120 \mathrm{~km}$ with the observed shape (Fig. 3). The fragments that penetrated below $27 \mathrm{~km}$ must have contributed to the damage in order to match the shockwave arrival times (SM section 2.4).

The number of houses damaged per 1000 inhabitants (table S11) (SM section 2.3) falls off with distance from the airburst source $(r)$ as $r^{-2.6 \pm 1.2}$, with overpressure calculated to fall off as $r^{-2.4}$ (fig. S39). In Chelyabinsk itself, 3613 apartment buildings (about 44\%) had shattered and broken glass, but these were not evenly distributed in the city (fig. S37). Sharp sounds heard after the shock wave also point to the fragmentation's causing a complicated distribution of pressure. Structural damage included the collapse of a zinc factory roof.

Directly below the fireball's path, the shock wave was strong enough to blow people off their feet. In Yemanzhelinsk, window frames facing the
Fig. 2. Fireball visual magnitude irradiance light curve, normalized to $100-\mathbf{k m}$ distance. The bold dashed line shows the model fit to the light curve (SM section 1.2), with thin lines showing total mass of all fragments passing a given altitude $(\mathrm{kg})$ and the altitude-dependent rate of energy deposition as a fraction of the original kinetic energy $\left(\mathrm{km}^{-1}\right)$.

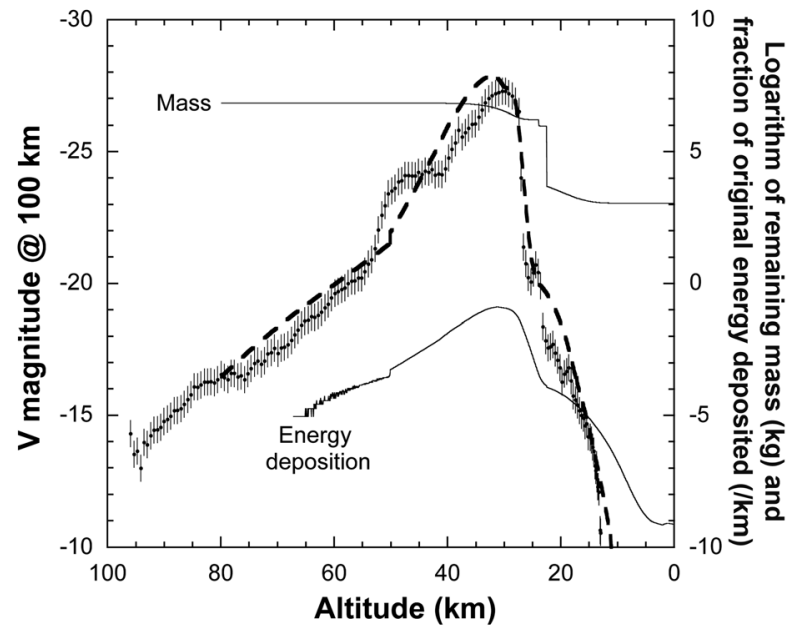

trajectory were pushed inwards, and suspended ceilings were sucked down above broken windows (fig. S36G). There was no structural damage to buildings, other than a statue of Pushkin inside the local library, cracked by a blown-out window frame. Cracks in walls were documented in nearby Baturinsky and Kalachevo.

Electrophonic sounds were heard (SM section 1.6), but there was no evidence of an electromagnetic pulse (EMP) under the track in neighboring Emanzhelinka. Due to shock-wave-induced vibrations, electricity and cell phone connectivity was briefly halted in the Kunashaksky district at the far northern end of the damage area. The gas supply was briefly interrupted in some districts because of valves reacting to the vibrations.

People found it painful to look at the bright fireball, but glancing away prevented lasting eye damage. Of 1113 respondents to an Internet survey who were outside at the time, 25 were sunburned $(2.2 \%), 315$ felt hot $(28 \%)$, and 415 (37\%) felt warm (SM section 2.2). Mild sunburns were reported throughout the survey area (table S7), reflecting the fact that ultraviolet (UV) flux density falls off as $\sim r^{-2}$. In Korkino, $30 \mathrm{~km}$ from the point of peak brightness, one resident reported getting a mild sunburn on the face, followed by peeling of skin. Such effects occur at a minimum erythema dose of $\sim 1000 \mathrm{~J} / \mathrm{m}^{2}$ (14) of 290- to 320-nm radiation (mostly UV-B). Assuming 6000-K radiation (9), the calculated dose would have been $\sim 200 \mathrm{~J} / \mathrm{m}^{2}$ at Korkino. Ground reflectance of UV light by snow may have further increased the dose.

Out of the total 1674 collected Internet responses, 374 mention 452 body injuries or inconveniences (SM section 2.2). Of those, 5.3\% reported sunburn, $48 \%$ eyes hurt, and $2.9 \%$ retinal burns. Because of the shock wave, $6.4 \%$ reported a concussion or mental confusion, upset, or exhaustion as a result of excessive stress. Flying glass and falling building debris affected a relatively small fraction of respondents: $4.8 \%$ reported cuts and $2.9 \%$ reported bruises, but no broken bones were reported.

The percentage of people asking for medical assistance (table S10) dropped with distance ac- cording to $r^{-3.2 \pm 0.5}$ (SM section 2.1). The majority of injuries (1210) took place in the densely populated Chelyabinsk city, but the highest fraction of people asking for assistance was near the trajectory track in the Korkinsky district $(0.16 \%)$.

\section{Meteorite Recovery}

Shock radiation contributed to surface heating and ablation but did not completely evaporate all fragments of Chelyabinsk, unlike in the case of Tunguska (3). Meteorites of $\sim 0.1 \mathrm{~g}$ fell near Aleksandrovka close to the point of peak brightness, masses of $\sim 100 \mathrm{~g}$ fell further along the trajectory near Deputatskiy, and at least one of $3.4 \mathrm{~kg}$ fell near Timiryazevskiy. One hit the roof of a house in Deputatskiy (fig. S46). Fallingsphere models suggest that they originated at 32to $26-\mathrm{km}$ altitude (fig. S52), where the meteor model shows rapid fragmentation (fig. S18C). The location of the meteorites is consistent with prevailing northwest winds of 5 to $15 \mathrm{~m} / \mathrm{s}$ (fig. S24). An estimated 3000 to $5000 \mathrm{~kg}$ fell in this area (SM section 3.1).

Two main fragments survived the disruption at $29.7 \mathrm{~km}$. They flared around $24 \mathrm{~km}$, with one falling apart at $18.5 \mathrm{~km}$ and the other remaining luminous down to $13.6 \mathrm{~km}$ (Fig. 1 and fig. S15). Light-curve modeling (SM section 1.2) suggests that from this material another $\sim 1$ ton in larger fragments up to 100 to $400 \mathrm{~kg}$ in mass reached the ground. A 7-m-sized hole was discovered in 70-cm-thick ice on Lake Chebarkul (fig. S53A), in line with the trajectory (SM section 1.1). A lakeshore video security camera, pointed to the site, recorded the impact (fig. S53B). Small meteorite fragments were recovered over an area up to $50 \mathrm{~m}$ from the impact location (Fig. 4C). Impact models (figs. S18 and S54) suggest a 200- to $1000-\mathrm{kg}$ meteorite would be required to create such a hole. A mass of $\geq 570 \mathrm{~kg}$ was recovered from the lake bed (fig. S53C).

The combined 4 to 6 tons of surviving meteorites is only 0.03 to $0.05 \%$ of the initial mass. Seventy-six percent of the meteoroid evaporated, with most of the remaining mass converted into 
Table 1. Atmospheric trajectory and pre-atmospheric orbit for the Chelyabinsk meteoroid, with $\mathbf{2}$ standard deviation uncertainties. Angular elements are for equinox ]2000.0.

\begin{tabular}{|c|c|c|c|c|}
\hline Atmospheric trajectory & Chelyabinsk & Pre-atmospheric orbit & Chelyabinsk & Itokawa \\
\hline$H_{\mathrm{b}}$ (beginning height, $\mathrm{km}$ ) & $97.1 \pm 1.6$ & $T_{\mathrm{J}}$ (Tisserand's parameter) & $3.87 \pm 0.24$ & 4.90 \\
\hline$H_{\mathrm{m}}$ (peak brightness, km) & $29.7 \pm 1.4$ & $a$ (semimajor axis, $\mathrm{AU})$ & $1.76 \pm 0.16$ & 1.324 \\
\hline$H_{\mathrm{f}}($ disruption, $\mathrm{km})$ & $27.0 \pm 1.4$ & $e$ (eccentricity) & $0.581 \pm 0.018$ & 0.280 \\
\hline$H_{\mathrm{e}}$ (end height, $\mathrm{km}$ ) & $13.6 \pm 1.4$ & $q$ (perihelion distance, $\mathrm{AU}$ ) & $0.739 \pm 0.020$ & 0.953 \\
\hline$V_{\infty}$ (entry speed, km/s) & $19.16 \pm 0.30$ & $\omega$ (argument of perihelion, ${ }^{\circ}$ ) & $108.3 \pm 3.8$ & 162.8 \\
\hline$h$ (entry elevation angle, ${ }^{\circ}$ ) & $18.3 \pm 0.4$ & $\Omega$ (longitude of ascending node, ${ }^{\circ}$ ) & $326.4422 \pm 0.0028$ & 69.1 \\
\hline$a_{\mathrm{z}}$ (entry azimuth angle from south, ${ }^{\circ}$ ) & $283.2 \pm 0.4$ & $i$ (inclination, ${ }^{\circ}$ ) & $4.93 \pm 0.48$ & 1.6 \\
\hline$V_{\mathrm{g}}$ (geocentric entry speed, $\mathrm{km} / \mathrm{s}$ ) & $15.3 \pm 0.4$ & $Q$ (aphelion distance, $\mathrm{AU}$ ) & $2.78 \pm 0.20$ & 1.70 \\
\hline $\mathrm{Ra}_{\mathrm{g}}$ (geocentric right ascension of radiant, ${ }^{\circ}$ ) & $333.2 \pm 1.6$ & $T_{\mathrm{p}}$ (perihelion time) & $2012-12-31.9 \pm 2.0$ & 2013-07-10.8 \\
\hline Dec $_{g}$ (geocentric declination of radiant, ${ }^{\circ}$ ) & $+0.3 \pm 1.8$ & Epoch (ET) & 2013-02-15.139 & 2013-04-18.0 \\
\hline
\end{tabular}

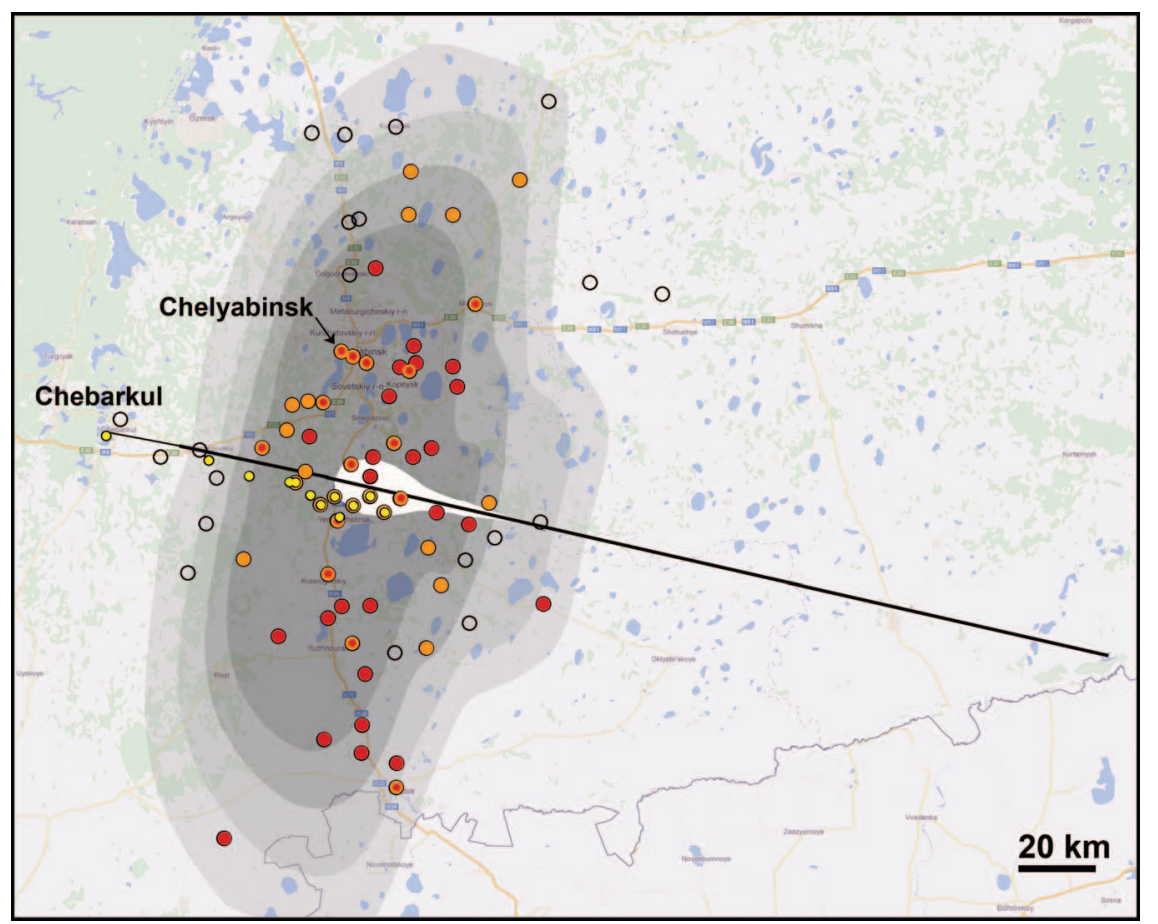

Fig. 3. Map of glass damage on the ground with models of overpressure. Field survey data are shown in solid orange circles for reported damage and open black circles for no damage; solid red circles show the most damaged villages in each district, as reported by the government. Each point, irrespective of population density, represents one of many villages or city districts scattered throughout the area. Model contours (with progressive gray scale) represent kinetic energies and overpressures from inside out: $300 \mathrm{kT} \Delta p>1000 \mathrm{~Pa}$, $520 \mathrm{kT} \Delta p>1000 \mathrm{~Pa}, 300 \mathrm{kT} \Delta p>500 \mathrm{~Pa}$, and $520 \mathrm{kT} \Delta p>500 \mathrm{~Pa}$, respectively. Also shown are the locations of meteorite finds (yellow points) and the ground-projected fireball trajectory (black line), moving from 97-km altitude on the right to $14-\mathrm{km}$ altitude on the left. White shows the fireball brightness on a linear scale.

dust (SM section 1.3). Witnesses reported smelling "sulfur" and burning odors over a wide region concentrated near the fireball trajectory, starting about an hour after the fireball and lasting through much of the day (SM section 1.5) (fig. S34).

\section{Characterization of Recovered Meteorites}

The unusually effective fragmentation and small surviving mass may have been caused by structural and material weakness. The asteroid had a lower compressive strength than the $\sim 330 \mathrm{MPa}$ measured for recovered, surviving meteorites (SM section S4.1). The light curve (Fig. 2) is modeled with fragmentation starting at a low $0.2 \mathrm{MPa}$ dynamic pressure but tolerating higher pressure with decreasing fragment size. This is similar to other meteorite falls, where initial weakness was attributed to macroscopic cracks or microscopic porosity (15). For Chelyabinsk, however, the physical weakness is not microporosity related. X-ray computed tomography (SM section 4.2) revealed a degree of compaction consistent with the lack of intragranular porosity typical of LL chondrites (16).

Some laboratory-broken meteorites fragmented along shock veins (fig. S55), a possible weakness in the material that could have contributed to the abundant dust formation. The meteorite is composed of a breccia (17) of mildly shocked lighter clasts and moderately shocked darker clasts with abundant thin to $\mathrm{cm}$-wide shock melt veins (Fig. 4A) (SM section 4.4). A peculiar feature is that some shock veins exhibit a metal layer located $\sim 20$ micrometers inside the vein, which follows the outer contours of the vein (Fig. 4B), indicating that metal initially segregated from the most rapidly solidifying rims of the vein. This could contribute to weakness. Metal-rich tendrils also project outward from the vein.

The mineral compositional ranges (SM section 4.4) are slightly larger than those reported before (18), but still compatible with a classification as LL5, shock stage S4 (19). The classification as LL chondrite is substantiated by oxygen and chromium isotope studies (SM section 4.5 to 4.7), which put the meteorite near the $\mathrm{L}$ end of the LL field $(20,21)$ (Fig. 4D and fig. S68). Iron content and oxidation state also support the LL chondrite classification (Fig. 4E and fig. S58). Rare earth element abundances are more similar to L chondrites (Fig. 4F and table S18), whereas one measuring reflectance spectrum better matches that of $\mathrm{H}$ chondrites (fig. S72).

The Chelyabinsk (LL) parent body experienced a substantial thermal and/or collision resetting event $115 \pm 21$ million years after the formation of the solar system (25), not experienced by most other LL chondrites, possibly due to a major impact event near its site of origin on the parent body. The phosphate $\mathrm{U}-\mathrm{Pb}$ age is $4452 \pm 21$ million years (SM section 4.8) (fig. S70), much younger than the majority of other ordinary chondrite phosphate ages dated by conventional thermal ionization mass spectrometry methods $(22,23)$. Perhaps one other piece of evidence for this is the $4.48 \pm 0.12$ billion years $\mathrm{Pb}-\mathrm{Pb}$ isochron age of phosphates in a granitelike fragment found in the LL3 to LL6 chondrite regolith breccia Adzhi-Bogdo (24), an observed fall in Mongolia in 1949.

Chelyabinsk shows a common orientation of metal grains indicating an impact-related petrofabric (fig. S59), stronger than that seen in any other ordinary chondrite of any shock stage (26) (fig. S60). This petrofabric probably reflects the most recent extraterrestrial shock event experienced by the 
Chelyabinsk meteoroid. The magnetic susceptibility value is at the upper end of the range for LL type (fig. S61) (27), closer to L-type chondrites, suggestive of higher metal content in Chelyabinsk than a typical LL chondrite. However, detailed analysis of the remanent magnetization suggests that a shock event or the conditions of atmospheric entry led to substantial resetting of the remanence (SM section 4.3).

Recent meteoroid heating events are normally recorded by thermoluminescence (TL) (SM section 4.10). In this case, the induced TL level of Chelyabinsk is lower than other petrographic type 5 or 6 chondrites, possibly because shock metamorphism to the level of S4 (30 to $35 \mathrm{GPa}$ ) (fig. S79) destroyed feldspar, the mineral phase responsible for the TL signal (28). Atmospheric heating did not cause loss of natural TL signal, the steep thermal gradient being consistent with a very thin fusion crust on the measured samples (29). The natural TL value is consistent with the meteoroid's having been heated at a perihelion distance of 0.6 to 0.8 astronomical units (AU) (Table 1).

The shock did not remove all organic matter from the meteorite. Methanol-soluble polar organic compounds (SM section 4.11) were detected in impact melt vein and chondrite fractions using electrospray ionization ion cyclotron resonance Fourier-Transform mass spectrometry (30). Out of more than 18,000 resolved mass peaks, 2536 could be assigned to compounds containing $\mathrm{C}$, $\mathrm{H}, \mathrm{N}, \mathrm{O}$, or $\mathrm{S}$. The organic signature is typical of other shocked LL chondrites, showing a higher abundance of oxygen and nitrogen atoms in the impact melt (fig. S83). The presence of oxygenated sulfur is indicated by CHOS compounds containing on average three more oxygen atoms than $\mathrm{CHO}$ and $\mathrm{CHNO}$ compounds. The high abundance of CHOS compounds in a homologous series across the entire mass range testifies that most of these did not result from terrestrial contamination.

Impact shock-induced fracturing on the Chelyabinsk parent body was followed by melting of metal and sulfides, which are pressuredriven through the cracks. There are cases in which this increased a meteorite's mechanical strength, the residual heat facilitating the process. However, in the case of Chelyabinsk, the production of cracks weakened the meteorite material more than shock melting increased its strength.

\section{Source and Evolution of the Chelyabinsk Meteoroid}

Chelyabinsk adds an LL5 type meteorite to a short list (SM section 1.1) of 18 different types of meteorites with known pre-atmospheric orbits (1). Only $8.2 \%$ of falls are LL chondrites (31). The Chelyabinsk meteorite is of particular interest because it is of the same type as asteroid Itokawa, from which samples were collected by the Hayabusa Mission (32). Indeed, both Itokawa and Chelyabinsk have similar low-inclined lowsemimajor axis orbits (Table 1), which, according to one model (33), imply a $62 \%, 11 \%$, and $25 \%$ probability for Chelyabinsk (and $71 \%, 0 \%$, and $29 \%$ probability for Itokawa) of originating from the secular $v_{6}$ resonance, the 3:1 mean-motion resonance, and the Intermediate Mars Crosser region, respectively. Multiplying these probabilities, assuming all LL chondrites enter the near-Earth object region through the same escape route, there is now an $86 \%$ probability that they originated from $v_{6}$. This supports the hypothesis (34) that they originated from the inner part of the LL-type (35) Flora asteroid family, which straddles the $v_{6}$ resonance in $1.6^{\circ}$ to $7.7^{\circ}$ inclined orbits (36).

As a group, LL chondrites have a cosmic ray exposure age peaking at $\sim 17$ million years (34). Chelyabinsk, on the other hand, was exposed only since $\sim 1.2$ million years ago (SM section 4.12 ). The responsible breakup that first exposed the Chelyabinsk meteoroid surface to cosmic rays was not likely part of the ongoing collision cascade in
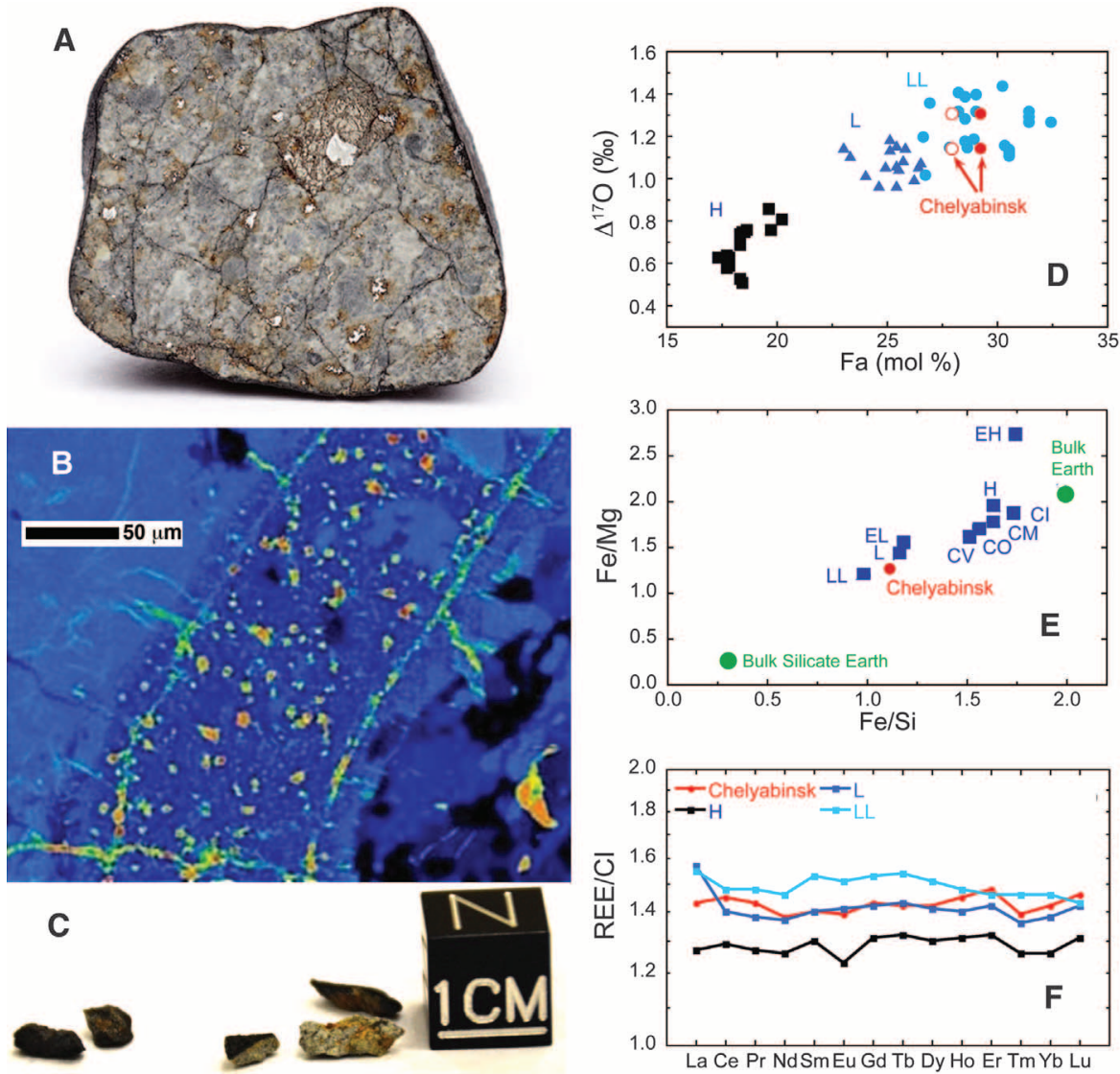

Fig. 4. Meteorite material properties, chemical and isotopic compositions. (A) Chelyabinsk meteorite (diameter $\sim 4 \mathrm{~cm}$ ) showing shock veins. (B) Fe element map of a shock vein. Note the metal layer (shown in green) located $\sim 20$ micrometers inside the vein. (C) Meteorite fragments recovered from the icecovered lake Chebarkul. (D) $\Delta^{17} 0$ versus Fa molar percent of olivine in Chelyabinsk, compared with other ordinary chondrites of type $\mathrm{H}, \mathrm{L}$, and $\mathrm{LL}(38)$. Fa is defined as molar ratio of $\mathrm{Fe} /(\mathrm{Fe}+\mathrm{Mg})$ in olivine. Average $\Delta^{17} 0$ values from the University of New Mexico (UNM) [1.15 \pm 0.06 per mil (\%o)] and from the Korea Polar Research Institute (KOPRI) $(1.31 \pm 0.04 \%$ o) for Chelyabinsk likely reflect indigenous heterogeneity in oxygen isotopes. Solid symbols are Fa number measured in this study (SM section S4.3), whereas open symbols are from (18). (E) Ratio plots of the three major elements ( $\mathrm{Mg}$, Si, Fe; together with oxygen $>90 \%$ of mass) for Chelyabinsk and the main chondrite groups. The bulk Earth and bulk silicate Earth compositions were taken from (39), chondrite compositions from (40). (F) Cl chondrite normalized rare earth elemental pattern of Chelyabinsk compared with the average chondrite group compositions of $(38,41)$. 


\section{References and Notes}

1. P. Jenniskens et al., Science 338, 1583-1587 (2012)

2. M. B. E. Boslough, D. A. Crawford, Ann. N.Y. Acad. Sci. 822, 236-282 (1997).

3. V. V. Svetsov, V. V. Shuvalov, in Catastrophic Events Caused by Cosmic Objects, V. Adushkin, I. Nemtchinov, Eds. (Springer, Dordrecht, Netherlands, 2008), pp. 227-267.

4. D. R. Christie, P. Campus, in Infrasound Monitoring for Atmospheric Studies, A. Le Pichon, E. Blanc, A. Hauchecorne, Eds. (Springer, Dordrecht, Netherlands, 2010), pp. 29-75.

5. D. Yeomans, P. Chodas, Additional Details on the Large Fireball Event over Russia on Feb. 15, 2013. NASA NEO Program Office Announcement, March 1, 2013 (NASA, Washington, DC, 2013).

6. Materials and methods are available as supporting material on Science Online.

7. E. Tagliaferri, R. Spalding, C. Jacobs, S. P. Worden, A. Erlich, in Hazards Due to Comets and Asteroids, Space Science Series, T. Gehrels, M. S. Matthews. A. Schumann, Eds., (Univ. of Arizona Press, Tucson, AZ, 1994), pp. 199-220.

8. T. A. Ens, P. G. Brown, W. N. Edwards, E. A. Silber, J. Atmos. Sol. Terr. Phys. 80, 208-229 (2012)

9. I. V. Nemtchinov et al., Icarus 130, 259-274 (1997)

10. D. O. Revelle, Z. Ceplecha, ESA Special Publ. 495, 507-512 (2001)

11. J. Borovicka, P. Spurny, L. Shrbeny, Trajectory and orbit of the Chelyabinsk superbolide. CBET 3423, Central Bureau for Astronomical Telegrams, International Astronomical Union (2013); www.icq.eps.harvard.edu/ CBET3423.html

12. J. Zinn, J. Drummond, JGR Space Physics 110, A04306 (2005).

13. S. Glasstone, P. ]. Dolan, The Effects of Nuclear Weapons, Third Edition (U.S. Government Printing Office, Washington, DC, 1977)

14. M.-W. Huang, P.-Y. Lo, K.-S. Cheng, EURASIP J. Adv. Signal Process. 2010, 483562 (2010).

15. O. Popova et al., Meteorit. Planet. Sci. 46, 1525-1550 (2011).
16. G. J. Consolmagno, D. T. Britt, R. J. Macke, Chem. Erde 68, 1-29 (2008)

17. A. Bischoff, E. R. D. Scott, K. Metzler, C. A. Goodrich, in Meteorites and the Early Solar System II, D. S. Lauretta, H. Y. McSween Jr., Eds. (Univ. of Arizona Press, Tucson, AZ, 2006), pp. 679-712.

18. M. A. Nazarov, D. D. Badyukov, N. N. Kononkova, I. V. Kubrakova, Chelyabinsk Meteoritical Bulletin: Entry for Chelyabinsk; http://www.lpi.usra.edu/meteor/metbull. php?code $=57165$ (2013)

19. D. Stöffler, K. Keil, E. R. D. Scott, Geochim. Cosmochim. Acta 55, 3845-3867 (1991).

20. R. N. Clayton, T. K. Mayeda, ]. N. Goswami, E. ]. Olsen, Geochim. Cosmochim. Acta 55, 2317-2337 (1991).

21. A. Trinquier, J.-L. Birck, C. J. Allègre, Astrophys. J. 655, 1179-1185 (2007).

22. C. Göpel, G. Manhès, C. J. Allègre, Earth Planet. Sci. Lett. 121, 153-171 (1994)

23. Y. Amelin, Science 310, 839-841 (2005).

24. K. Terada, A. Bischoff, Astrophys. J. 699, L68-L71 (2009).

25. ]. N. Connelly et al., Science 338, 651-655 (2012)

26. J. M. Friedrich, ]. C. Bridges, M.-S. Wang, M. E. Lipschutz, Geochim. Cosmochim. Acta 68, 2889-2904 (2004).

27. P. Rochette et al., Meteorit. Planet. Sci. 38, 251-268 (2003).

28. C. P. Hartmetz, D. W. G. Sears, Meteoritics 22, 400-401 (1988)

29. D. W. Sears, A. A. Mills, Nat. Phys. Sci (London) 242, 25-26 (1973).

30. P. Schmitt-Kopplin et al., Proc. Natl. Acad. Sci. U.S.A. 107, 2763-2768 (2010)

31. T. L. Dunn, T. H. Burbine, W. F. Bottke Jr., ]. P. Clark, Icarus 222, 273-282 (2013).

32. T. Nakamura et al., Science 333, 1113-1116 (2011).

33. W. F. Bottke et al. Icarus 156, 339-433 (2000).

34. P. Michel, M. Yoshikawa, Astron. Astrophys. 449, 817-820 (2006).

35. V. Reddy et al., Icarus 216, 184-197 (2011).

36. D. Nesvorny, A. Morbidelli, D. Vokrouhlicky, W. F. Bottke, M. Broz, Icarus 157, 155-172 (2002).
37. E. Schunová et al., Icarus 220, 1050-1063 (2012).

38. ]. Troiano, D. Rumble III, M. L. Rivers, ]. M. Friedrich, Geochim. Cosmochim. Acta 75, 6511-6519 (2011).

39. W. F. McDonough, in The Mantle and Core, Vol. 2, Treatise on Geochemistry, R. W. Carlson, Ed. (Elsevier-Pergamon, Oxford, 2003), pp. 547-568.

40. ]. T. Wasson, G. W. Kallemeyn, Phil. Trans. R. Soc London A 325, 535-544 (1988).

41. J. M. Friedrich, M.-S. Wang, M. E. Lipschutz, Geochim Cosmochim. Acta 67, 2467-2479 (2003).

Acknowledgments: The Russian Academy of Sciences (RAS) field study of the Chelyabinsk airburst was supported by the Institute for Dynamics of Geospheres and grants of the Federal Targeted Program Scientific and Educational Human Resources of Innovation-Driven Russia and the RAS Presidium Program Fundamental Problems of Investigation and Exploration of the Solar System. The office of Chelyabinsk Oblast Governor Mikhail Yurevich provided assistance. S. Petukhov and I. Talyukin from the Universe History Museum in Dedovsk contributed samples, as did M. Boslough of Sandia National Laboratories. U. Johann (Astrium Satellites $\mathrm{GmbH}$ ) calculated the Chebarkul hole position from Pléiades $1 \mathrm{~A}$ satellite observations. D. F. Blake provided use of a petrographic microscope. P.]. acknowledges support from the NASA Near Earth Object Observation Program, Q.Z.Y. and M.E.Z. from the NASA Cosmochemistry Program, and M.G. from the Academy of Finland.

\section{Supplementary Materials}

www.sciencemag.org/content/342/6162/1069/suppl/DC1

Supplementary Text

Figs. $\mathrm{S} 1$ to $\mathrm{S} 87$

Tables S1 to S24

References (42-157)

1 July 2013; accepted 28 October 2013

Published online 7 November 2013;

10.1126/science. 1242642

\section{Nanoscale $\mathrm{Fe}_{2} \mathrm{O}_{3}$-Based Catalysts for Selective Hydrogenation of Nitroarenes to Anilines}

\author{
Rajenahally V. Jagadeesh, ${ }^{1}$ Annette-Enrica Surkus, ${ }^{1}$ Henrik Junge, ${ }^{1}$ \\ Marga-Martina Pohl, ${ }^{1}$ jörg Radnik, ${ }^{1}$ ]abor Rabeah, ${ }^{1}$ Heming Huan, ${ }^{2}$ \\ Volker Schünemann, ${ }^{2}$ Angelika Brückner, ${ }^{1}$ Matthias Beller ${ }^{1 *}$
}

Production of anilines - key intermediates for the fine chemical, agrochemical, and pharmaceutical industries-relies on precious metal catalysts that selectively hydrogenate aryl nitro groups in the presence of other easily reducible functionalities. Herein, we report convenient and stable iron oxide $\left(\mathrm{Fe}_{2} \mathrm{O}_{3}\right)$-based catalysts as a more earth-abundant alternative for this transformation. Pyrolysis of iron-phenanthroline complexes on carbon furnishes a unique structure in which the active $\mathrm{Fe}_{2} \mathrm{O}_{3}$ particles are surrounded by a nitrogen-doped carbon layer. Highly selective hydrogenation of numerous structurally diverse nitroarenes (more than 80 examples) proceeded in good to excellent yield under industrially viable conditions.

$\mathrm{B}$ eginning in the $1950 \mathrm{~s}$, the development of organometallic catalysts proceeded to revolutionize organic synthesis at scales

${ }^{1}$ Leibniz-Institut für Katalyse e.V. an der Universität Rostock, Albert-Einstein Strasse 29a, D-18059 Rostock, Germany ${ }^{2}$ Technische Universität Kaiserslautern, Fachbereich Physik, ErwinSchrödinger-Strasse 46, D-67663 Kaiserslautern, Germany.

*Corresponding author. E-mail: matthias.beller@catalysis.de ranging from the laboratory bench to the industrial manufacture of fine and bulk chemicals. This success was mainly due to the use of noble metal complexes - for example, palladium, rhodium, ruthenium, and iridium (1). However, the high price and limited availability of these precious metals (2) have spurred interest in catalysis with more earth-abundant alternatives, especially iron (3-8).
The durability and copious supply of iron salts coupled with their environmentally benign nature and low toxicity make them ideal catalysts. Recently, structurally well-defined molecular iron complexes have been applied successfully in contexts where previously noble metals were required (9-19). However, most of these homogeneous complexes are rather sensitive and/or incorporate sophisticated (and thus synthetically demanding) ligand systems. In contrast, heterogeneous iron oxides are extremely stable and can be easily recycled. Important applications of heterogeneous iron catalysts include the production of olefins through the Fischer-Tropsch process $(20,21)$ and the hydrogenation of $\mathrm{CO}$ (22). Unfortunately, these known catalysts work under drastic conditions $\left(>275^{\circ} \mathrm{C}\right)$ and are therefore of limited use for the refinement of more complex substrates. In this context, we report special iron oxide-based catalysts that allow for a general and highly selective hydrogenation of nitroarenes under comparably mild conditions. The resulting anilines constitute key building blocks for the synthesis of fine (agrochemicals, dyes, pigments, and pharmaceuticals) as well as bulk chemicals (polymers) $(23,24)$. In particular, anilines used in life science applications are often structurally complex molecules decorated with diverse functional groups. Thus, achieving high chemoselectivity is of crucial importance for 